\title{
Identification of microRNAs associated with medullary thyroid carcinoma by bioinformatics analyses
}

\author{
XIANGJUN FU ${ }^{1,2}$, JUGAO FANG $^{1}$, MENG LIAN ${ }^{1}$, QI ZHONG ${ }^{1}$, \\ HONGZHI MA ${ }^{1}$, LING FENG ${ }^{1}$, RU WANG ${ }^{1}$ and HAIZHOU WANG ${ }^{1}$ \\ ${ }^{1}$ Department of Otorhinolaryngology Head and Neck Surgery, Beijing Tongren Hospital, \\ Capital Medical University, Beijing 100730; ${ }^{2}$ Department of Otorhinolaryngology Head and Neck Surgery, \\ Guangdong No. 2 Provincial People's Hospital, Guangzhou, Guangdong 510317, P.R. China
}

Received January 28, 2016; Accepted January 19, 2017

DOI: $10.3892 / \mathrm{mmr} .2017 .6547$

\begin{abstract}
The present study aimed to investigate the microRNA (miRNA) profile in human medullary thyroid carcinoma (MTC) tissue. The GSE40807 data profile was downloaded from the Gene Expression Omnibus database. Following preprocessing, differentially expressed microRNAs (DEMs) between MTC and healthy tissues were identified. Based on the obtained DEMs, transcription factor (TF)-miRNA and miRNA-target gene regulatory association pairs were predicted. Finally, functional enrichment analysis was performed on target genes of DEMs. Fifteen upregulated and 17 downregulated DEMs were identified. In the constructed TF-miRNA regulatory network, hsa-miR-9-5p was regulated by 9 TFs and hsa-miR-1 was regulated by 8 TFs. TFs of nuclear factor of $\kappa$ light polypeptide gene enhancer in B-cells $1(\mathrm{NF}-\kappa \mathrm{B} 1)$ and v-myc avian myelocytomatosis viral oncogene homolog (MYC) regulated 4 and 3 DEMs, respectively. In the miRNA-target gene regulatory network, hsa-miR-1, hsa-miR-9-5p, hsa-miR-96-5p and hsa-miR-590-5p were most upregulated. The target genes of these 4 miRNAs were primarily enriched in the mitogen activated protein kinase (MAPK) signaling pathway. Therefore, MAPK signaling pathway may serve important roles in MTC progression. In conclusion, the DEMs hsa-miR-1 and hsa-miR-9-5p, and TFs of NF- $\kappa$ B1 and MYC may be used as biomarkers for the diagnosis and treatment of MTC.
\end{abstract}

\section{Introduction}

Thyroid cancer is the most common type of cancer of the endocrine system, with cases increasing worldwide $(1,2)$.

Correspondence to: Dr Jugao Fang, Department of Otorhinolaryngology Head and Neck Surgery, Beijing Tongren Hospital, Capital Medical University, 8 Chongwenmennei Street, Dongcheng, Beijing 100730, P.R. China

E-mail: jugaofangfff@hotmail.com

Key words: medullary thyroid carcinoma, differentially expressed microRNAs, transcription factor, target gene
Thyroid cancer may be classified into numerous types according to the histopathological characteristics. Medullary thyroid carcinoma (MTC) is a form of thyroid cancer which originates from the parafollicular cells of the thyroid (3). It is the third most common type of thyroid cancer and accounts for $\sim 3 \%$ of all thyroid cancer cases. Approximately 1 in 4 of MTC cases are caused by mutations in the rearranged during transfection (RET) proto-oncogene (4). The majority of MTC cases are sporadic, presenting with metastatic disease at diagnosis (5). Nearly all patients with distant metastases succumb to this disease (6).

Presently, RET mutation have been suggested to be an indicator of the poor prognosis of MTC; however, this is not sufficient for understanding the underlying molecular mechanisms of MTC tumourigenesis. Soh et al (7) demonstrated that vascular endothelial growth factor receptor 2 was involved in the pathogenesis of MTC via promotion of pro-invasive and pro-angiogenic phenotypes. Additionally, dysregulation of the Dickkopf/Wnt signaling pathway inhibitor 4 has been identified in MTC (8). A previous study revealed that aberrant expression levels of microRNAs (miRNAs) have a potential role in tumourigenesis (9), which may provide novel insight in MTC research. Notably, increasing evidence has supported the important role of miRNAs in cancers including thyroid cancer $(10,11)$. For example, He et al (12) reported that three miRNAs, including miR-221, -222, and -146, are overexpressed in papillary thyroid cancer. Furthermore, miR-197 and -346 are significantly overexpressed in follicular thyroid cancers (13). Although great advances have been made in understanding the functions of miRNAs in thyroid cancers, the underlying molecular mechanisms of this disease remain to be elucidated.

The present study aimed to use GSE40807 miRNA microarray data provided by Lassalle et al (14) to identify differentially expressed miRNAs (DEMs) between human MTC and healthy control tissues. Subsequently, transcription factor (TF)-miRNA and miRNA-target gene regulatory networks were constructed. Finally, the target genes of DEMs were performed functional enrichment analyses to predict their potential functions that may be associated with MTC. To the best of our knowledge, this is the first time that the dataset of GSE40807 was analyzed. 


\section{Materials and methods}

Data source. The GSE40807 miRNA microarray dataset was downloaded from the Gene Expression Omnibus (www .ncbi.nlm.nih.gov/geo/) database in the National Center for Biotechnology Information based on the Agilent-019118 Human miRNA Microarray 2.0 G4470B platform (Agilent Technologies, Inc., Santa Clara, CA, USA). The dataset included 14 pairs of miRNA microarrays from human MTC and adjacent healthy tissues.

Data preprocessing and DEM analysis. The original data were firstly converted into identifiable expression form using a Linear Models for Microarray Data (limma) package in R language (http://www.bioconductor. org/packages/release/bioc/html/limma.html) (15). Following this, background correction and quartile data normalization were performed using a robust multiarray average algorithm affy package in R (http://www.bioconductor. org/packages/release/bioc/html/affy.html) (16).

TF-miRNA regulatory association pair prediction. The TF-miRNA regulatory database (TransmiR; cmbi.bjmu. edu.cn/transmir) (17) is a valuable resource for the study of TF-miRNA regulation, which provides an interface for easy retrieval of TF-miRNA regulatory pairs by searching for a miRNA or a TF. Currently, TransmiR has curated 735 entries, which includes 201 TFs, 209 miRNAs and 16 organisms from 268 publications. The present study inputted the obtained DEMs into the database and extracted regulatory association pairs between DEMs and TFs.

miRNA-target gene regulatory association pair prediction and TF-miRNA-target gene regulatory network construction. The starBase v2.0 (starbase.sysu.edu.cn/) database (18) provides certain miRNA-target regulatory association pairs which are verified by experiments and predicted by five algorithms including TargetScan (19), miRanda (20), Pictar2 (21), PITA (22) and RNA22 (23). In the present study, miRNA-target gene regulatory association pairs verified by $\geq$ one experiment and predicted by $\geq$ three algorithms were selected for construction of the regulatory network.

Based on the predicted TF-miRNA and miRNA-target gene regulatory association pairs, the TF-miRNA-target gene regulatory network was constructed using Cytoscape software version 3.2.0 (www.cytoscape.org/) (24). From the network, TFs, miRNAs and target genes that had higher connective degrees (hub nodes) were extracted. Hub nodes are small numbers of nodes with numerous interaction partners, which serve important roles in the network (25). Thus, these TFs, miRNAs and target genes might serve roles in MTC.

Functional enrichment analyses. clusterProfiler software (Bioconductor version 3.1; bioconductor.org/packages/release/bioc/html/clusterProfiler.html) (26) is a package used for gene classification and enrichment analysis. The Kyoto Encyclopedia of Genes and Genomes (KEGG; www .genome.ad.jp/kegg/) (27) is a database of biological systems that collects genomic, chemical and systemic functional information. To analyzed the potential biological functions of
Table I. Up- and downregulated miRs.

\begin{tabular}{lcl}
\hline miR & $\log _{2} \mathrm{FC}$ & \multicolumn{1}{c}{$\mathrm{P}$} \\
\hline hsa-miR-9-5p & 1.771272789 & $9.96 \mathrm{E}-05$ \\
hsa-miR-149-5p & -1.285205715 & 0.000830471 \\
hsa-miR-708-5p & -1.14714938 & 0.003865191 \\
hsa-miR-335-5p & 2.093096976 & 0.003949694 \\
hsa-miR-592 & 1.802025779 & 0.004011795 \\
hsa-miR-875-5p & -1.148677692 & 0.004350708 \\
hsa-miR-455-5p & -1.191505453 & 0.004403134 \\
hsa-miR-590-5p & 1.642054145 & 0.004446886 \\
hsa-miR-96-5p & 2.177657366 & 0.005246821 \\
hsa-miR-584-5p & -1.157995671 & 0.005083235 \\
hsa-miR-922 & 1.022980071 & 0.005377546 \\
hsa-miR-1 & 1.816907981 & 0.005578293 \\
hsa-miR-296-5p & -1.335624531 & 0.00595956 \\
hsa-miR-634 & -1.222619212 & 0.006085225 \\
hsa-miR-224-5p & 1.794623266 & 0.006202874 \\
hsa-miR-185-5p & 1.573515208 & 0.006464766 \\
hsa-miR-628-5p & 1.035790796 & 0.007467156 \\
hsa-miR-924 & -1.075200672 & 0.007467315 \\
hsa-miR-154-5p & 1.230953928 & 0.007599707 \\
hsa-miR-625-5p & 1.081907512 & 0.009088003 \\
hsa-miR-145-3p & 1.656652903 & 0.00054755 \\
hsa-miR-195-3p & -1.337668541 & 0.001551485 \\
hsa-let-7f-1-3p & -1.15398965 & 0.001765947 \\
hsa-miR-515-3p & -1.033833078 & 0.002354703 \\
hsa-miR-9-3p & 1.917564249 & 0.004292166 \\
hsa-miR-7-2-3p & -1.106382457 & 0.005054543 \\
hsa-miR-143-3p & 1.498076967 & 0.005687211 \\
hsa-miR-887-3p & -1.372273521 & 0.005784544 \\
hsa-miR-512-3p & -1.149225488 & 0.006533263 \\
hsa-miR-1237-3p & -1.009014763 & 0.006904609 \\
hsa-miR-371a-3p & -1.099403781 & 0.007869777 \\
hsa-let-7b-3p & -1.362383564 & 0.008104953 \\
\hline & & \\
\hline
\end{tabular}

$\log _{2} \mathrm{FC}>0$, upregulation; $\log _{2} \mathrm{FC}<0$, downregulation. miR; microRNA.

DEMs, KEGG pathway enrichment analysis was performed for the target genes of the obtained DEMs based on the clusterProfiler package. $\mathrm{P}<0.01$ was set as the threshold value.

Statistical analysis. DEMs between MTC and healthy tissues were identified using the limma (15) package (Bioconductor version 3.1). Student's t-test in the limma package was used to compare DEM values, and fold changes (FCs) were calculated. miRNAs with $\mathrm{P}<0.01$ and $\log _{2} \mathrm{FCl} \geq 1$ were selected as DEMs. $\mathrm{P}<0.05$ was considered to indicate a statistically significant difference.

\section{Results}

Identification of DEMs. A total of 32 DEMs were identified between MTC and healthy tissues. Among these DEMs, 15 were upregulated and 17 were downregulated (Table I). 

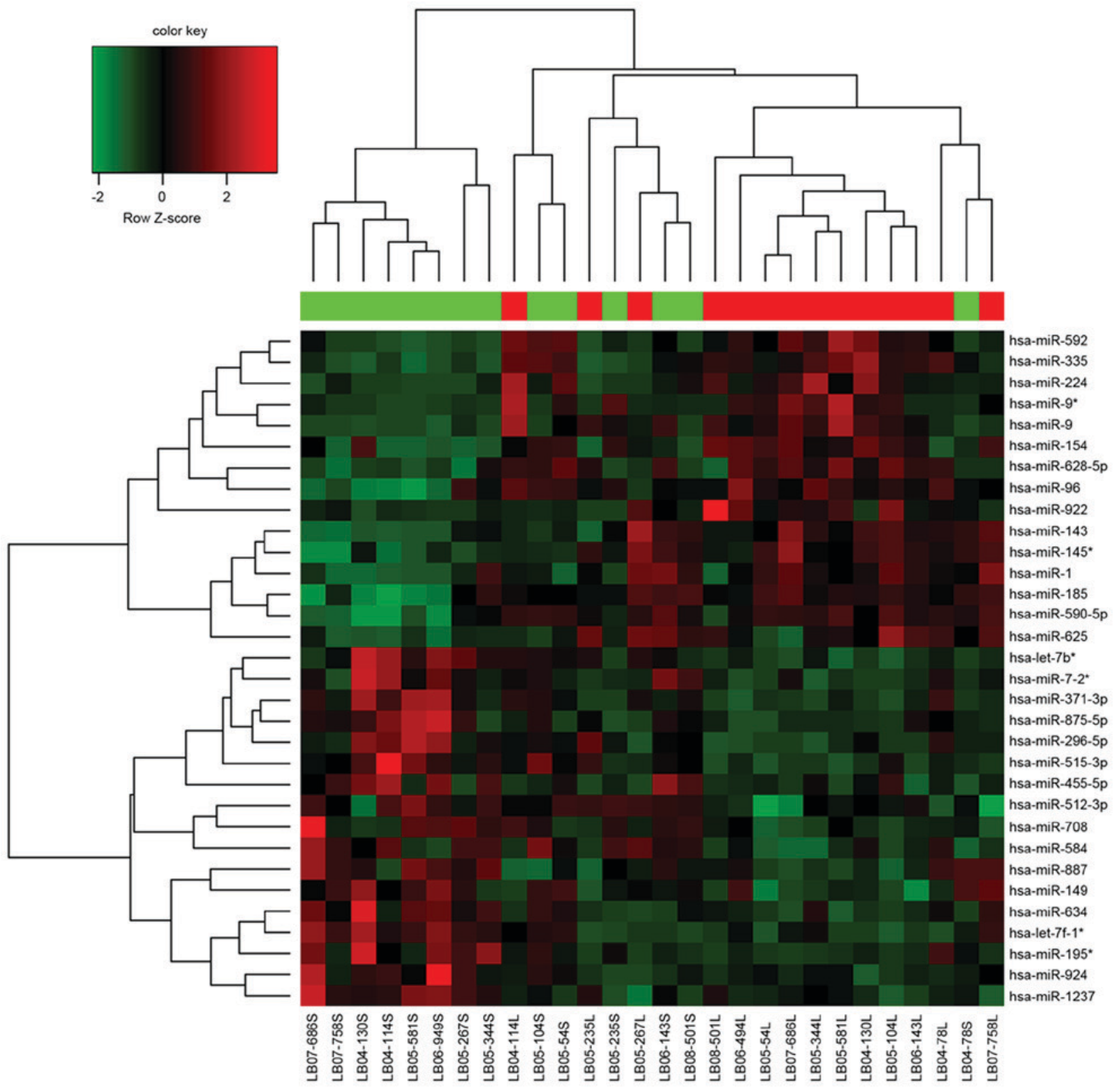

Figure 1. Heatmap of differentially expressed miRs. The upper color bar represents sample classes; red represents disease samples and green represents healthy controls. miR, microRNA.

Hierarchical clustering analysis of these DEMs and samples are presented in Fig. 1.

TF-miRNA regulatory relationship pairs. From TransmiR, 54 TF-miRNA regulatory association pairs were extracted, including 33 TFs and 10 miRNAs. Among these miRNAs, hsa-miR-9-5p was regulated by nine TFs, including nuclear factor of $\kappa$ light polypeptide gene enhancer in B-cells 1 (NF-kB1), and interleukin $1 \beta$ (IL-1 $\beta$ ), and hsa-miR-1 was regulated by eight TFs, including CCAAT/enhancer binding protein $\alpha$. Additionally, TFs of NF- $\kappa$ B1 regulated four DEMs, including hsa-miR-9-5p and -3p, and v-myc avian myelocytomatosis viral oncogene homolog (MYC) regulated three DEMs including hsa-miR-195-3p, hsa-let-7b-3p and hsa-let-7f-1-3p.

miRNA-target gene regulatory association pairs and TF-miRNA-target gene regulatory network construction.
From starBase, 1654 miRNA-target gene regulatory association pairs were obtained, including 12 DEMs and 1338 target genes. Among the 12 DEMs, hsa-miR-1, hsa-miR-9-5p, hsa-miR-96-5p and hsa-miR-590-5p had the top four highest connective degrees (feature miRNAs). Additionally, seven target genes that were regulated by at least four DEMs were identified (Table II).

Furthermore, based on the constructed miRNA-target gene and TF-miRNA regulatory networks, a TF-miRNAtarget gene regulatory network was constructed using Cytoscape software. In the network, there were 1654 miRNA-target gene and 54 TF-miRNA regulatory relationship pairs (Fig. 2).

Functional enrichment analyses. Among the 12 DEMs in the miRNA-target gene regulatory network, the target genes of hsa-miR-1, hsa-miR-9-5p, hsa-miR-96-5p and hsa-miR-590-5p 
Table II. Feature miRNAs and genes in the miR-target gene regulatory network.

\begin{tabular}{lr} 
Node & Number \\
\hline hsa-miR-9-5p & 326 \\
hsa-miR-96-5p & 297 \\
hsa-miR-1 & 246 \\
hsa-miR-590-5p & 146 \\
CRIM1 & 5 \\
KIF1B & 4 \\
NR4A3 & 4 \\
RNF111 & 4 \\
TNPO1 & 4 \\
FNDC3B & 4 \\
BCL11A & 4 \\
\hline
\end{tabular}

miR, microRNA; CRIM1, cysteine rich transmembrane bone morphogenic protein regulator 1; KIF1B, kinesin family member 1B; NR4A3, nuclear receptor subfamily 4 group A member 3; RNF111, ring finger protein 111; TNPO1, transportin-1; FNDC3B, fibronectin type III domain containing 3B; BCL11A, B-cell lymphoma/leukemia 11A.

were demonstrated to be enriched in the KEGG pathways, including the mitogen activated protein kinase (MAPK) signaling pathway, pathways in cancer, and during focal adhesion (Table III).

\section{Discussion}

Patients with progressive MTC have limited treatment options (28). Thus, understanding the underlying molecular mechanism of carcinogenesis may facilitate diagnosis and therapy options of this disease. In the present study, 15 upregulated and 17 downregulated DEMs were identified. In the constructed TF-miRNA regulatory network, hsa-miR-9-5p was regulated by 9 TFs and hsa-miR-1 was regulated by 8 TFs. The TFs of NF- $\mathrm{kB} 1$ and MYC regulated 4 and 3 DEMs, respectively. Additionally, the above two miRNAs served key roles in the miRNA-target gene regulatory network. Their target genes were primarily enriched in the MAPK signaling pathway and during focal adhesion. These miRNAs and signaling pathways may be important biomarkers for MTC diagnosis and treatment.

In the miRNA-target gene regulatory network, hsa-miR-1 was upregulated, and its target genes, including MAPK1, were enriched in numerous signaling pathways associated with cancer, including MAPK. MAPKs are a family of protein kinases whose functions are conserved during evolution from unicellular organisms (29). The MAPK signaling pathway consists of numerous key signaling components and phosphorylation events which control multiple fundamental cell processes including proliferation, differentiation and apoptosis (30). This signaling pathway has been frequently identified in activated in human cancers, which leads to malignant phenotypes including autonomous cell proliferation (31). Notably, Zatelli et al (32) demonstrated that the
Table III. Enriched signaling pathways involving differentially expressed miRs.

\begin{tabular}{ll}
\hline miR & \multicolumn{1}{c}{ Pathway description } \\
\hline hsa-miR-1 & Neurotrophin signaling pathway \\
& Renal cell carcinoma \\
& Axon guidance \\
& MAPK signaling pathway \\
& Pathways in cancer \\
& Neurotrophin signaling pathway \\
hsa-miR-9-5p & Bacterial invasion of epithelial cells \\
& Focal adhesion \\
& MAPK signaling pathway \\
& Endocytosis \\
& GnRH signaling pathway \\
hsa-miR-96-5p & ErbB signaling pathway \\
& Prostate cancer \\
& Neurotrophin signaling pathway \\
& Axon guidance \\
& MAPK signaling pathway
\end{tabular}

miR, microRNA; MAPK, mitogen activated protein kinase; GnRH, gonadotrophin-releasing hormone; ErbB, receptor tyrosine-protein kinase ERBB2.

growth of the TT MTC cell line depends on activation of the MAPK signaling pathway, which suggests its role in MTC. In addition, MAPK signaling is important in regulating cytokine signaling pathways (33). Cytokines are released in response to inflammation and immunity, and have important roles in cancer development and progression (34). It has been reported that undifferentiated thyroid cancer cells secrete cytokines (35). Taken together, the MAPK signaling pathway may serve important roles in MTC via hsa-miR-1 and its target gene MAPK1.

In addition to hsa-miR-1, hsa-miR-9-5p upregulated in the miRNA-target gene regulatory network, and was regulated by $9 \mathrm{TFs}$ in the TF-miRNA regulatory network, including NF- $\kappa B 1$ and IL-1ß. NF- $\kappa B$ is a transcription regulator activated by various intra- and extracellular stimuli. A previous study demonstrated that NF- $\mathrm{\kappa B} 1$ regulates the expression of genes involved in numerous processes, including proliferation and apoptosis (36). Inappropriate activation of NF- $\mathrm{\kappa B}$ has been associated with numerous inflammatory diseases, whereas persistent inhibition of NF- $\mathrm{KB}$ may lead to delayed cell growth (37). NF- $\mathrm{kB}$ has been demonstrated to be associated with the development of colorectal (38), breast (39), bladder (40), prostate (41) and advanced thyroid (36) cancers. On the other hand, IL-1 $\beta$, a member of the IL-1 cytokine family, is an important mediator of the inflammatory response. A previous study reported that inflammation is a critical component of tumor progression (42). Zeki et al (43) suggested that IL-1 regulates G1 cell cycle progression and arrest in papillary thyroid carcinoma cells. Therefore, NF- $\kappa \mathrm{B} 1, \mathrm{IL}-1 \beta$ and their regulated DEM hsa-miR-9-5p may serve important roles in MTC progression. 


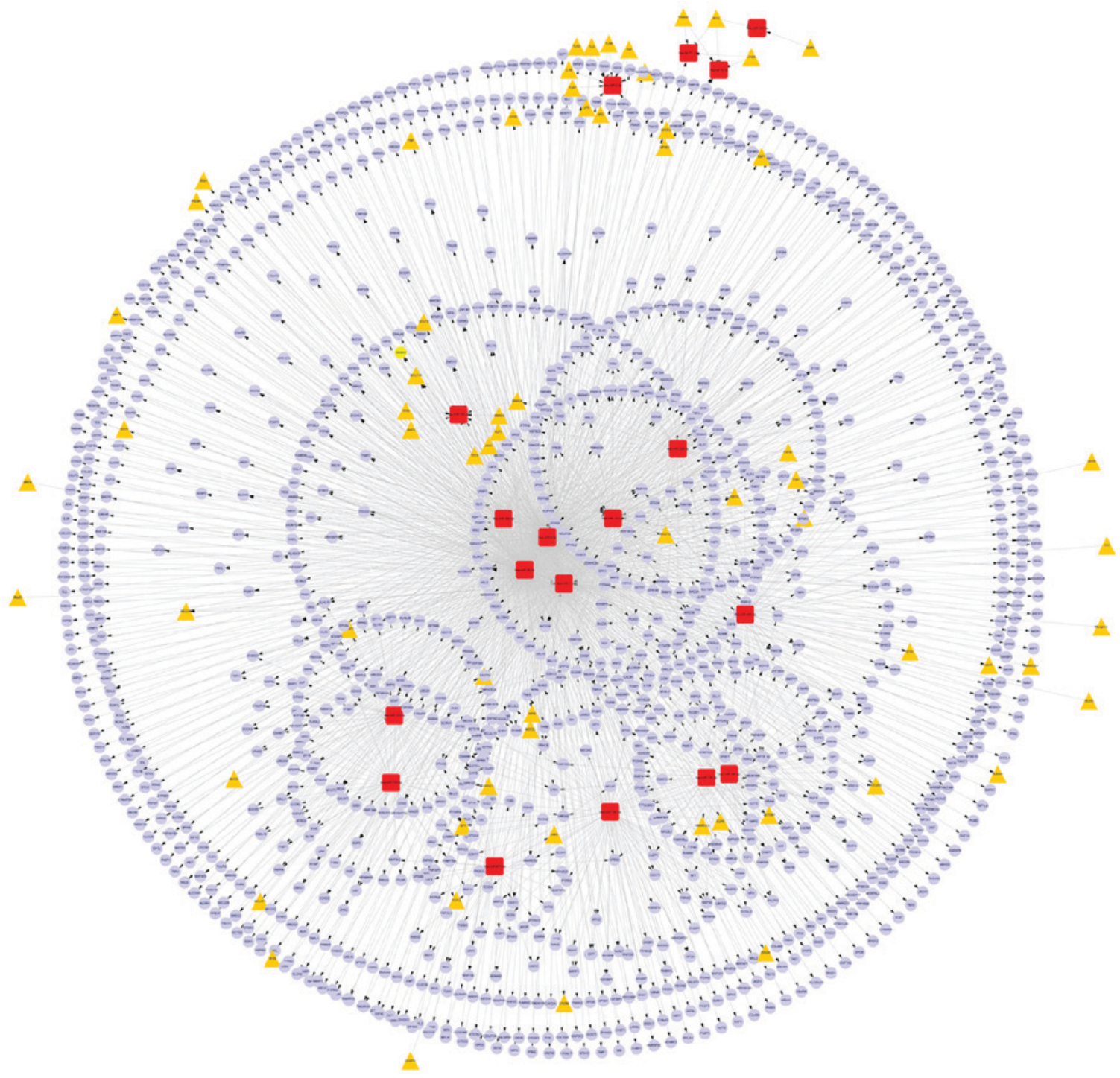

Figure 2. TF-DEM-Target comprehensive regulatory network. Red squares represent microRNAs, orange triangles represent TF genes and blue circles represent non-TF genes. TF, transcription factor; DEM, differentially expressed microRNA.

Additionally, TFs of MYC were demonstrated to regulate 3 DEMs including hsa-miR-195-3p, hsa-let-7b-3p and hsa-let-7f-1-3p. MYC is a multifunctional, nuclear phosphoprotein which serves roles in cell cycle progression, apoptosis and cellular transformation (44). The MYC gene has been widely implicated in numerous human cancers $(45,46)$. Khosla et al (47) revealed that MYC mRNA expression levels increased in apoptotic TT cells, suggesting its role in MTC. Its regulated miRNA hsa-miR-195-3p has been identified to be abnormally expressed in a variety of cancers. For example, levels were upregulated in breast cancer and downregulated in gastric, hepatocellular and bladder cancers (48-50). The roles of hsa-let-7b-3p and hsa-let-7f-1-3p in cancer remain to be elucidated; thus, it was hypothesized that these miRNAs may be involved in MTC via regulation from the $M Y C$ gene. Taken together, MYC and its regulated DEMs, hsa-miR-195-3p, hsa-let-7b-3p and hsa-let-7f-1-3p, may serve important roles in the development of MTC.

The present study identified numerous key miRNAs and TFs that may be associated with MTC using comprehensive bioinformatics methods. However, no experiments with tissues or cells were performed to validate the expression levels of these miRNAs and TFs; a key limitation of this study. Additionally, there were only 14 pairs of miRNA microarrays in the dataset. Further studies with experimental validations and more samples are required to validate these observations.

In conclusion, the results of the present study indicated that the DEMs hsa-miR-1, hsa-miR-9-5p and hsa-miR-195-3p may have the potential to be used as diagnostic and therapeutic targets of MTC. Additionally, hsa-miR-1 and its target gene MAPK1 may serve a role in MTC, involving in MAPK signaling pathway. Additionally, TFs of IL-1ß and MYC may be implicated in the development of MTC.

\section{Acknowledgements}

The present study was supported by the Beijing Municipal Science \& Technology Commission (grant no.Z141107002514003), the Beijing Municipal Administration of Hospitals Clinical Medicine Development of Special Funding Support (grant no. XMLX201311) and the National Natural Science Foundation of China (grant no. 81473499). 


\section{References}

1. He W, Qi B, Zhou Q, Lu C, Huang Q, Xian L and Chen M: Key genes and pathways in thyroid cancer based on gene set enrichment analysis. Oncol Rep 30: 1391-1397, 2013.

2. Geraldo MV and Kimura ET: Integrated analysis of thyroid cancer public datasets reveals role of post-transcriptional regulation on tumor progression by targeting of immune system mediators. PLoS One 10: e0141726, 2015.

3. Hu MI, Vassilopoulou-Sellin R, Lustig R and Lamont JP: Thyroid and parathyroid cancers. Cancer Management: A Multidisciplinary Approach 11: 2008.

4. Lodish MB and Stratakis CA: RET oncogene in MEN2, MEN2B, MTC and other forms of thyroid cancer. Expert Rev Anticancer Ther 8: 625-632, 2008.

5. Santarpia L, Calin GA, Adam L, Ye L, Fusco A, Giunti S, Thaller C, Paladini L, Zhang X, Jimenez C, et al: A miRNA signature associated with human metastatic medullary thyroid carcinoma. Endocr Relat Cancer 20: 809-823, 2013.

6. Hofstra R, Stelwagen T, Stulp RP, de Jong D, Hulsbeek M, Kamsteeg EJ, van den Berg A, Landsvater RM, Vermey A, Molenaar WM, et al: Extensive mutation scanning of RET in sporadic medullary thyroid carcinoma and of RET and VHL in sporadic pheochromocytoma reveals involvement of these genes in only a minority of cases. J Clin Endocrinol Metab 81: 2881-2884, 1996.

7. Soh EY, Duh QY, Sobhi SA, Young DM, Epstein HD, Wong MG, Garcia YK, Min YD, Grossman RF, Siperstein AE and Clark OH: Vascular endothelial growth factor expression is higher in differentiated thyroid cancer than in normal or benign thyroid. J Clin Endocrinol Metab 82: 3741-3747, 1997.

8. Maliszewska A, Leandro-Garcia LJ, Castelblanco E, Macià A, de Cubas A, Goméz-López G, Inglada-Pérez L, Álvarez-Escolá C, De la Vega L, Letón R, et al: Differential gene expression of medullary thyroid carcinoma reveals specific markers associated with genetic conditions. Am J Pathol 182: 350-362, 2013

9. Iorio MV and Croce CM: MicroRNA dysregulation in cancer: Diagnostics, monitoring and therapeutics. A comprehensive review. EMBO Mol Med 4: 143-159, 2012.

10. Pallante P, Visone R, Croce CM and Fusco A: Deregulation of microRNA expression in follicular cell-derived human thyroid carcinomas. Endocr Relat Cancer 17: F91-F104, 2010.

11. Visone R, Pallante P, Vecchione A, Cirombella R, Ferracin M, Ferraro A, Volinia S, Coluzzi S, Leone V, Borbone E, et al: Specific microRNAs are downregulated in human thyroid anaplastic carcinomas. Oncogene 26: 7590-7595, 2007.

12. He H, Jazdzewski KW, Li W, Liyanarachchi S, Nagy R, Volinia S, Calin GA, Liu CG, Franssila K, Suster S, et al: The role of microRNA genes in papillary thyroid carcinoma. Proc Natl Acad Sci USA 102: 19075-19080, 2005.

13. Weber F, Teresi RE, Broelsch CE, Frilling A and Eng C: A limited set of human microRNA is deregulated in follicular thyroid carcinoma. J Clin Endocrinol Metab 91: 3584-3591, 2006.

14. Lassalle S, Zangari J, Popa A, Ilie M, Hofman V, Long E, Patey M, Tissier F, Belléannée $\mathrm{G}$, Trouette $\mathrm{H}$, et al: MicroRNA-375/SEC23A as biomarkers of the in vitro efficacy of vandetanib. Oncotarget 7: 30461-30478, 2016.

15. Smyth GK: Limma: linear models for microarray data. In: Bioinformatics and computational biology solutions using $\mathrm{R}$ and Bioconductor. Springer, New York, NY, pp397-420, 2005.

16. Gautier L, Cope L, Bolstad BM and Irizarry RA: affy-analysis of Affymetrix GeneChip data at the probe level. Bioinformatics 20 : 307-315, 2004.

17. Wang J, Lu M, Qiu C and Cui Q: TransmiR: A transcription factor-microRNA regulation database. Nucleic Acids Res 38 (Database issue): D119-D122, 2010.

18. Li JH, Liu S, Zhou H, Qu LH and Yang JH: starBase v2. 0: Decoding miRNA-ceRNA, miRNA-ncRNA and protein-RNA interaction networks from large-scale CLIP-Seq data. Nucleic Acids Res 42 (Database issue): D92-D97, 2014.

19. Lewis BP, Shih IH, Jones-Rhoades MW, Bartel DP and Burge CB: Prediction of mammalian microRNA targets. Cell 115: 787-798, 2003.

20. John B, Enright AJ, Aravin A, Tuschl T, Sander C and Marks DS: Human microRNA targets. PLoS Biol 2: e363, 2004.

21. Krek A, Grün D, Poy MN, Wolf R, Rosenberg L, Epstein EJ, MacMenamin P, da Piedade I, Gunsalus KC, Stoffel M and Rajewsky N: Combinatorial microRNA target predictions. Nat Genet 37: 495-500, 2005.
22. Kertesz M, Iovino N, Unnerstall U, Gaul U and Segal E: The role of site accessibility in microRNA target recognition. Nat Genet 39: 1278-1284, 2007.

23. Ritchie W, Flamant S and Rasko JE: Predicting microRNA targets and functions: Traps for the unwary. Nat Methods 6: 397-398, 2009

24. Shannon P, Markiel A, Ozier O, Baliga NS, Wang JT, Ramage D, Amin N, Schwikowski B and Ideker T: Cytoscape: A software environment for integrated models of biomolecular interaction networks. Genome Res 13: 2498-2504, 2003.

25. He $X$ and Zhang J: Why do hubs tend to be essential in protein networks? PLoS Genet 2: e88, 2006.

26. Yu G, Wang LG, Han Y and He QY: clusterProfiler: An R package for comparing biological themes among gene clusters. OMICS 16: 284-287, 2012.

27. Kanehisa M and Goto S: KEGG: Kyoto encyclopedia of genes and genomes. Nucleic Acids Res 28: 27-30, 2000.

28. Elisei R, Schlumberger MJ, Müller SP, Schöffski P, Brose MS, Shah MH, Licitra L, Jarzab B, Medvedev V, Kreissl MC, et al: Cabozantinib in progressive medullary thyroid cancer. J Clin Oncol 31: 3639-3646, 2013

29. Johnson GL and Lapadat R: Mitogen-activated protein kinase pathways mediated by ERK, JNK and p38 protein kinases. Science 298: 1911-1912, 2002.

30. Dhillon A, Hagan S, Rath O and Kolch W: MAP kinase signalling pathways in cancer. Oncogene 26: 3279-3290, 2007.

31. Bradham C and McClay DR: p38 MAPK in development and cancer. Cell Cycle 5: 824-828, 2006.

32. Zatelli MC, Piccin D, Tagliati F, Bottoni A, Luchin A and degli Uberti EC: SRC homology-2-containing protein tyrosine phosphatase-1 restrains cell proliferation in human medullary thyroid carcinoma. Endocrinology 146: 2692-2698, 2005.

33. Sumimoto $H$, Imabayashi $F$, Iwata $T$ and Kawakami $Y$ : The BRAF-MAPK signaling pathway is essential for cancer-immune evasion in human melanoma cells. J Exp Med 203: 1651-1656, 2006.

34. Dranoff G: Cytokines in cancer pathogenesis and cancer therapy. Nat Rev Cancer 4: 11-22, 2004.

35. Fiore L, Pollina LE, Fontanini G, Casalone R, Berlingieri MT, Giannini R, Pacini F, Miccoli P, Toniolo A, Fusco A and Basolo F: Cytokine production by a new undifferentiated human thyroid carcinoma cell line, FB-1. J Clin Endocrinol Metab 82: 4094-4100, 1997.

36. Dolcet X, Llobet D, Pallares J and Matias-Guiu X: NF-kB in development and progression of human cancer. Virchows Arch 446: 475-482, 2005.

37. Caamano J and Hunter CA: NF-kappaB family of transcription factors: Central regulators of innate and adaptive immune functions. Clin Microbiol Rev 15: 414-429, 2002.

38. Lewander A, Butchi AK, Gao J, He LJ, Lindblom A, Arbman G, Carstensen J, Zhang ZY and Sun XF; Swedish Low-Risk Colorectal Cancer Study Group: Polymorphism in the promoter region of the NFKB1 gene increases the risk of sporadic colorectal cancer in Swedish but not in Chinese populations. Scand J Gastroenterol 42: 1332-1338, 2007.

39. Curran JE, Weinstein SR and Griffiths LR: Polymorphic variants of NFKB1 and its inhibitory protein NFKBIA and their involvement in sporadic breast cancer. Cancer Lett 188: 103-107, 2002.

40. Riemann K, Becker L, Struwe H, Rübben H, Eisenhardt A and Siffert W: Insertion/deletion polymorphism in the promoter of NFKB1 as a potential molecular marker for the risk of recurrence in superficial bladder cancer. Int J Clin Pharmacol Ther 45: 423-430, 2007.

41. Zhang P, Wei Q, Li X, Wang K, Zeng H, Bu H and Li H: A functional insertion/deletion polymorphism in the promoter region of the NFKB1 gene increases susceptibility for prostate cancer. Cancer Genet Cytogenet 191: 73-77, 2009.

42. Coussens LM and Werb Z: Inflammation and cancer. Nature 420: 860-867, 2002

43. Zeki K, Morimoto I, Arao T, Eto S and Yamashita U: Interleukin-1alpha regulates $\mathrm{G} 1$ cell cycle progression and arrest in thyroid carcinoma cell lines NIM1 and NPA. J Endocrinol 160: 67-73, 1999.

44. Hoffman B and Liebermann D: Apoptotic signaling by c-MYC. Oncogene 27: 6462-6472, 2008.

45. Ellwood-Yen K, Graeber TG, Wongvipat J, Iruela-Arispe ML, Zhang J, Matusik R, Thomas GV and Sawyers CL: Myc-driven murine prostate cancer shares molecular features with human prostate tumors. Cancer Cell 4: 223-238, 2003. 
46. Ma L, Young J, Prabhala H, Pan E, Mestdagh P, Muth D Teruya-Feldstein J, Reinhardt F, Onder TT, Valastyan S, et al: miR-9, a MYC/MYCN-activated microRNA, regulates E-cadherin and cancer metastasis. Nat Cell Biol 12: 247-256, 2010.

47. Khosla S, Oursler M, Schroeder M and Eberhardt N: Transforming growth factor-beta 1 induces growth inhibition of a human medullary thyroid carcinoma cell line despite an increase in steady state c-myc messenger ribonucleic acid levels. Endocrinology 135: 1887-1893, 1994.

48. Heneghan HM, Miller N, Lowery AJ, Sweeney KJ, Newell J and Kerin MJ: Circulating microRNAs as novel minimally invasive biomarkers for breast cancer. Ann Surg 251: 499-505, 2010.
49. Takamizawa J, Konishi H, Yanagisawa K, Tomida S, Osada H Endoh H, Harano T, Yatabe Y, Nagino M, Nimura Y, et al: Reduced expression of the let-7 microRNAs in human lung cancers in association with shortened postoperative survival. Cancer Res 64: 3753-3756, 2004.

50. Xu T, Zhu Y, Xiong Y, Ge YY, Yun JP and Zhuang SM: MicroRNA-195 suppresses tumorigenicity and regulates G1/S transition of human hepatocellular carcinoma cells. Hepatology 50: 113-121, 2009. 